\section{B1 IS SUPER ACCELERATED HEPATITIS B VACCINATION BEST WAY FORWARD?}

doi:10.1136/sextrans-2012-050601d.1

1J Thomas, ${ }^{2}{ }^{2} \mathrm{~S}$ Busby, ${ }^{2} \mathrm{M}$ Chauhan. ${ }^{1}$ Royal Liverpool University Hospital, Liverpool, UK; ${ }^{2}$ Sunderland Royal Hospital, Sunderland, UK

Background Hepatitis B vaccination offers protection against hepatitis B in high-risk individuals like IVDU, homosexuals and in sex workers. Super accelerated vaccination schedule of $0,7,21$ days has been found to increase the compliance to the vaccination and has been found to be effective against hepatitis B transmission. We followed this schedule in our GUM clinic.

Objectives 1 . To assess the uptake of Hepatitis B vaccination in homosexual and bisexual men. 2. To assess whether full STI screening including testing for HIV, hepatitis B and C is being done along with checking $\mathrm{HBs}$ antibody levels in those already immunised.

Methods We looked at 30 patient notes, which included homosexual and bisexual men.

Results All (100\%) patients got full STI screen including HIV, hepatitis $\mathrm{B}$ and $\mathrm{C}$ testing. $30 \%$ of patients were vaccinated before and so immune to hepatitis B as shown by HBs antibody levels. Out of the remaining $70 \%$ of patients, $40 \%$ received three doses of Hepatitis B vaccination on the super accelerated vaccination schedule. $13 \%$ got two doses, $10 \%$ got only one dose and the remaining $7 \%$ declined.

Conclusion All high risk individuals attending the sexual health clinic should receive a full STI screen including HIV, Hepatitis B, C testing and $\mathrm{HBs}$ antibody levels checked who has previously received vaccination. The accelerated vaccination schedule encourages patients to receive the full course of immunisation. Education of highrisk individuals is necessary to improve the vaccination uptake. A written card with vaccination appointment dates given to patients at the beginning will further improve the patient attendance to finish the full course of the vaccination.

\section{B2 1 SPACE OCCUPYING LESION IN AN HIV POSITIVE MALE- ARE COMMON THINGS ALWAYS COMMON?}

doi:10.1136/sextrans-2012-050601d.2

${ }^{1} \mathrm{G}$ Haidari, ${ }^{*} \mathrm{C}$ S Singh, ${ }^{1} \mathrm{~B}$ Freudenthal, ${ }^{1} \mathrm{~B}$ Peters, ${ }^{1} \mathrm{R}$ Kulasegaram, ${ }^{1} \mathrm{M}$ Desai, ${ }^{2} \mathrm{~B}$ Bodi. ${ }^{1}$ St Thomas' Hospital; ${ }^{2}$ Kings College Hospital, London, UK

A 62-year-old man with well controlled HIV infection presented to clinic in December 2011, with a 6 week history of increasing forgetfulness, unsteady gait and visual disturbance. He was well established on highly active antiretroviral therapy, with a CD4 count of 524 and an undetectable viral load. On examination his GCS was $15 / 15$, and he was noted to have a homonymous hemianopia. MRI confirmed a large mass in the deep thalamus extending into the brainstem which did not typically enhance. He went on to have a brain biopsy confirming a high grade glioblastoma multiforme. Post biopsy he developed a dense right sided hemiplegia thought to be due to disease progression. There have been 21 case reports of glioblastoma multiforme in HIV, raising the possibility we are now seeing an increasing number in HIV positive patients. In all documented cases, HIV was not found in glial tumours, although it is thought the role of the immune system in glial cell transformation could be compromised in HIV.
Discussion HIV patients with glioblastoma multiforme are presenting at younger ages and have poorer outcomes than non-HIV patients. Glioblastoma multiforme should be considered in the differential for HIV patients presenting with neurological symptoms, especially in patients stable on highly active antiretroviral therapy with a good CD4 count, in an effort to reduce diagnostic delay.

\section{B3 $\quad$ SOCIAL AND WELFARE IN CLINIC SUPPORT SERVICE}

doi:10.1136/sextrans-2012-050601d.3

J Hale.* First Point Access and Assessment Worker

Background Patients social and welfare care needs can be poorly served. Two factors contributed to this, lack of a specialised staff member for social and welfare needs and the few external organisation that are known, frequently changed their criteria or closed down.

Aim Provide stable background for patient's thus decreasing nonadherence, enabling more free appointment time for increasing number of new diagnosis.

Method Employ an experienced worker, to complete the multidisciplinary holistic care for people living with HIV, ensuring joined-up care between medical and social well-being with an excellent two way communication. We have one set of notes for all these needs and one focal point for external services to feedback to. Patients receive full assessment in addition to their presenting issue.

During an assessment, advice and referrals are offered on:

- Social/Peer Support Groups

- Benefits/Housing: assess patient's entitlement, avoiding unnecessary supporting medical letters. Alleviates patients approaching different legal orgs with same request. This system ensures who do require the help receive it.

- Immigration

- Employment skills and voluntary work

- Travelling abroad with HIV

- Insurance

- HIV at work

- Complimentary therapies

- Recreational drug misuse

- Domestic violence

- Sex workers

- Charity goods

Results and Conclusions 350 patients seen, $1 \mathrm{~h}$ each per year. Service currently just 2 days per week Full time role is envisaged. Database used to produce outcomes including needs, referrals made and gaps in services. Clinic staff freed to spend time on medical issues.

B4 IN PID

doi:10.1136/sextrans-2012-050601d.4

A de Burgh-Thomas, ${ }^{*}$ A Hills, V Medland. Gloucestershire Care Services, Gloucestershire Royal Hospital, Gloucestershire, UK

Objectives To review the management of PID in hospital inpatients and compare it to standards of care. 
Methods We reviewed the management of all those women admitted to hospital in Gloucestershire over a calendar year who were coded as having PID. We compared their management against BASHH and the RCOG standards (see abstract B4 table 1).

Results 20 Patients were identified.

Conclusions There is insufficient liaison between Gynaecology and GUM. We will discuss simple measures that have been introduced and planned changes to improve the management of PID.
Abstract B4 Table 1 Compliance of management of patients with PID with standards

\begin{tabular}{lcc}
\hline & Target & Achieved \\
\hline Adequate sexual history & $95 \%$ & $25 \%$ \\
Chlamydia testing & $100 \%$ & $55 \%$ \\
Gonorrhoea testing & $100 \%$ & $70 \%$ \\
Appopriate antibiotics & $100 \%$ & $75 \%$ \\
GUM follow-up arranged & $95 \%$ & $5 \%$ \\
Contact tracing 6/12 pre & $60 \%$ & $0 \%$ \\
\hline
\end{tabular}

